\title{
A PROOF OF THE UNIFORMIZATION THEOREM FOR ARBITRARY PLANE DOMAINS
}

\author{
YUVAL FISHER, JOHN H. HUBBARD AND BEN.S. WITTNER
}

(Communicated by Irwin Kra)

\begin{abstract}
We present a simple constructive proof of the Uniformization Theorem which works for plane domains. The proof is a combination of covering space theory and Koebe's constructive proof of the Riemann mapping theorem, and the resulting algorithm can be used to estimate the Poincaré metric for the domain.
\end{abstract}

1. Introduction. In the study of iteration of rational functions [B], we constantly need the Poincaré metric for plane domains. The Uniformization Theorem is usually proved using potential theory. The proof is quite involved, and it is very hard to see how to compute the resulting metric.

We present here a simpler constructive proof which works for plane domains, or more generally any Riemann surface some covering space of which can be embedded in C. It is nothing but a combination of covering space theory and Koebe's constructive proof of the Riemann mapping theorem. The algorithm has been implemented in FORTRAN.

UNIFORMIZATION THEOREM FOR PLANE DOMAINS. If $U$ is a region contained in $\mathbf{C}-\{a, b\}$ for some $a \neq b$ in $\mathbf{C}$, then there exists a conformal isomorphism of $\tilde{U}$ with $D$.

2. Preliminaries. Let $D \subset \mathbf{C}$ be the open unit disk, $D_{r}$ be the open disk of radius $r$ centered at $O$, and $\bar{D}_{r}$ be the closure of $D_{r}$.

Let $m_{a}(z)=(z+a) /(1+\bar{a} z)$, where $\bar{a}$ is the complex conjugate of $a$. For $a \in D$, $m_{a}: D \rightarrow D$ is a conformal bijection with inverse $m_{-a}(z)$. Aut $(D)$, the group of analytic automorphisms of $D$, is precisely $\left\{e^{i \theta} m_{a}(z) \mid a \in D\right\}$.

Also define $\operatorname{sq}(z)=z^{2}$, and let $\tilde{U}$ denote the universal covering space of $U$.

2.1 The universal covering of $\mathbf{C}-\{0,1\}$. Let $H$ denote the upper half plane. The following is essentially proved by Theorem 7 and Theorem 8 of Ahlfors $[\mathbf{A}, \mathrm{p}$. 281].

THEOREM 2.1. The modular function $\lambda: H \rightarrow \mathbf{C}-\{0,1\}$ is a universal covering map.

2.2 Limits of analytic functions. The next theorem is stated in elementary texts for plane domains only, but the proof immediately generalizes to an arbitrary Riemann surface $\Omega$, at least if $\Omega$ is a countable union of compact subsets. Actually, a theorem of Radó asserts that all Riemann surfaces $\Omega$ have this property [F, p. 186], but this result is difficult. It is easy if $\Omega$ is a covering space of a plane domain.

Received by the editors February 25, 1986 and, in revised form, March 13, 1987.

1980 Mathematics Subject Classification (1985 Revision). Primary 30A46; Secondary 30F20. 
LEMMA 2.2. If $\Omega$ is a Riemann surface which is a covering space of a plane domain $U$, then $\Omega$ is a countable union of compact subsets.

PROOF. This follows from the fact that the fundamental group of $U$ is countable, which itself follows from the fact that $U$ is a countable union of open discs. Q.E.D.

REMARK. Notice that $\mathbf{C}-\left\{1, \frac{1}{2}, \frac{1}{3}, \ldots\right\}$ does not have a countable fundamental group; of course it is not an open subset of $\mathbf{C}$.

THEOREM 2.3(Montel). If $f_{n}: \Omega \rightarrow \mathbf{C}$ is a sequence of uniformly bounded analytic maps, then $f_{n}$ has a subsequence which converges uniformly on compact subsets.

PROOF. [A, p. 220].

LEMMA 2.4. If $p: U \rightarrow U_{0}$ is a covering map, $\pi: Y \rightarrow U_{0}$ continuous, and $f: U \rightarrow Y$ is an open surjective map such that the following diagram commutes, then $f$ is a covering map.

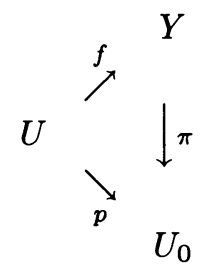

PROOF. This is straightforward.

\section{Proof of the Uniformization Theorem for plane domains.}

Part a: Reduction to bounded domains.

LEMMA 3.1. Without loss of generality we may assume that $0 \in U \subset D$.

ProOF. This is where we use Theorem 2.1. Let $p: D \rightarrow \mathrm{C}-\{a, b\}$ be a universal covering map; there is such a $p$ by Theorem 2.1 since $D$ is conformally isomorphic to $H$. Let $U_{0}$ be a connected component of $p^{-1}(U)$; then $U_{0}$ is contained in $D$, and since $U_{0}$ is a covering space of $U$ they have isomorphic universal covering spaces. The theorem will be proved if we can prove it for $U_{0}$. If $a$ is any point of $U_{0}$, we can further replace $U_{0}$ by its image under $m_{-a}$, so that $0 \in U_{0}$. Q.E.D.

REMARK. If $U$ is bounded to begin with we just need to scale it to bring it into the disc. More generally if any component $K$ of $\mathbf{C}-U$ is not a point, we can replace $U$ by its image under the conformal mapping $\overline{\mathbf{C}}-\bar{K} \rightarrow D$, where $\overline{\mathbf{C}}$ is the Riemann sphere and $\bar{K}$ is the closure of $K$ in $\overline{\mathbf{C}}$. But the case where $U$ is the complement of a Cantor set is of particular importance in the applications we have in mind; we do not know any way to reduce that case to bounded domains without using the modular function or something equivalent. The case where $U$ is the complement of a finite set is also of interest, specifically to the accessory parameter problem; it also seems to require the modular function.

Part b: The main construction. We will construct recursively a sequence of domains $U_{0}, U_{1}, \ldots, U_{n}$, with all $U_{n}$ contained in $D$. Moreover, each $U_{n}$ will come with a covering map $p_{n}: U_{n} \rightarrow U_{n-1}$, which will be of degree 1 or 2 . Thus each $U_{n}$ is a covering space of $U$, and there exist covering maps $f_{n}: \tilde{U} \rightarrow U_{n}$, which can also be thought of as a sequence of mappings $f_{n}: \tilde{U} \rightarrow D$. We will then prove that the $f_{n}$ converge to an isomorphism $f: \tilde{U} \rightarrow D$. 
Assume $U_{0}, \ldots, U_{n-1}$ have been constructed, with each $U_{i} \subset D$, and $0 \in U_{i}$ for each $i$. Further assume $p_{1}, \ldots, p_{n-1}$ have been constructed, and $p_{i}(0)=0$ for each $i$.

Let $a_{n} \in \partial U_{n-1}$ be a point on the boundary of $U_{n-1}$ that is closest to the origin, so that $D\left(0,\left|a_{n}\right|\right) \subset U_{n-1}$. Let $q_{n}: D \rightarrow D$ be given by

$$
q_{n}=m_{a_{n}} \circ \mathrm{sq} \circ m_{b_{n}},
$$

where $b_{n}=\sqrt{-a_{n}}$ is an arbitrary square root; note that $q_{n}(0)=0$.

There are now two cases to distinguish. See Figure 1.

Case 1. $a_{n}$ is in a noncompact component of $D-U_{n-1}$, i.e. on an "outer" component of the boundary of $U_{n-1}$. Then $q_{n}^{-1}\left(U_{n-1}\right)$ consists of two connected components, intersecting at $-b_{n}$. Choose the one containing zero, call it $U_{n}$, and let $p_{n}$ be the restriction of $q_{n}$ to it.

Case 2. $a_{n}$ is in a compact component of $D-U_{n-1}$, i.e. on an "inner" boundary component. This time $q_{n}^{-1}\left(U_{n-1}\right)$ is connected; call it $U_{n}$ and let $p_{n}$ be the restriction of $q_{n}$ to it.

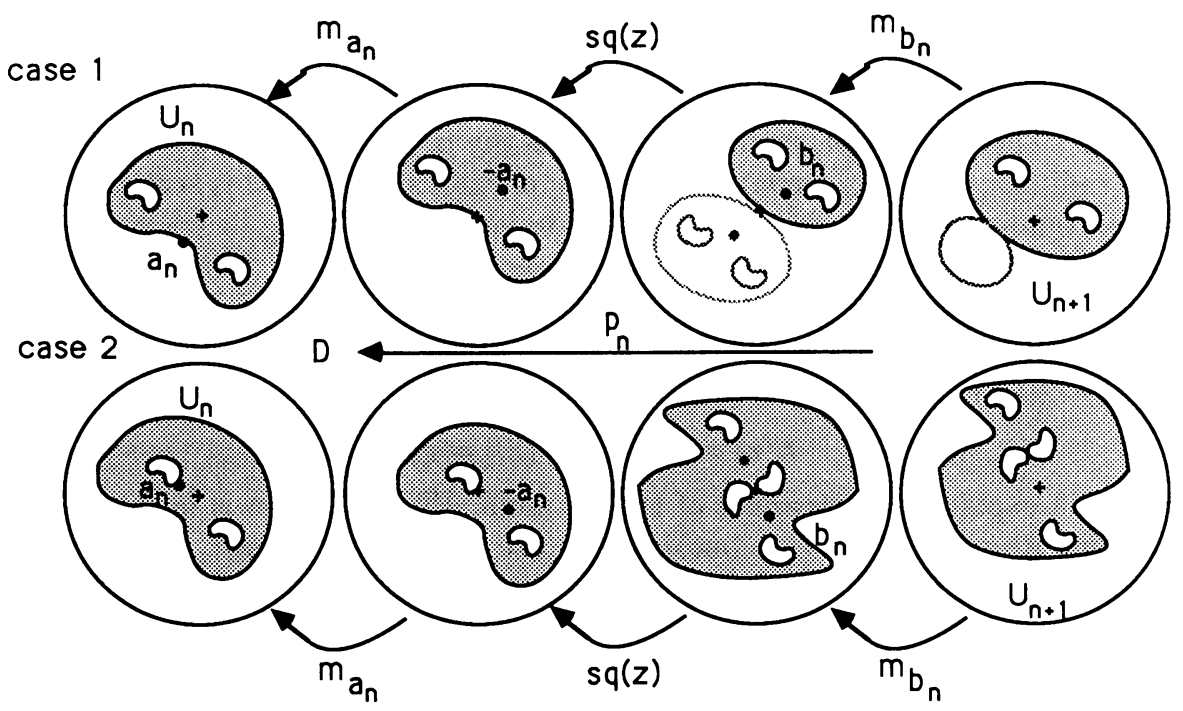

FIGURE 1

Part c: Proof of convergence. Choose $p: \tilde{U} \rightarrow U_{0}$ a universal cover, and let $\tilde{0}$ be an inverse image of 0 . Because the maps $p_{n}: U_{n} \rightarrow U_{n-1}$ are covering maps, there exist unique analytic mappings $f_{n}: \tilde{U} \rightarrow U_{n}$ with $f_{n}(\tilde{0})=0$ which make the diagram in Figure 2 commute.

The $f_{n}$ are uniformly bounded, so that $\left\{f_{n}\right\}$ is a normal family with a convergent subsequence converging to some analytic function $f$ (see 2.3).

Proposition 3.2. The mapping $f: \tilde{U} \rightarrow D$ is a covering map.

With this claim, the main theorem is proved. For then $\tilde{U}$ is a covering of $D$, and since $D$ is simply connected, any analytic covering of $D$ is an isomorphism.

PROOF. We will prove that $f$ is surjective first and then that it is a covering map of its image. 


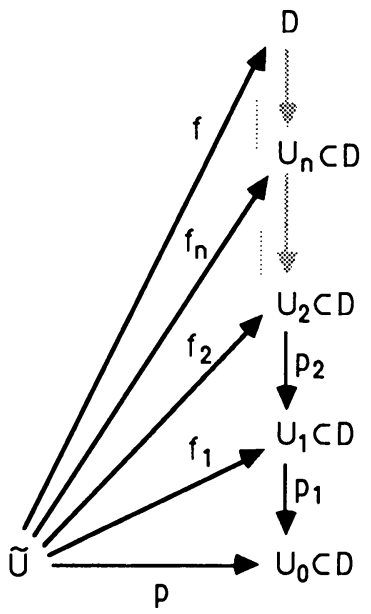

FIGURE 2

LEMMA 3.3. The mapping $f$ is surjective.

We will show that the domains $U_{n}$ contain larger and larger discs centered at the origin. The function $h$, defined below, has the property that if $U_{n}$ contains the disk of radius $r$, then $U_{n+1}$ contains the disc of radius $h(r)$. In this section, we will discuss $h$ qualitatively. In fact it is possible to compute $h(r)$ explicitly and use this computation to estimate the rate of convergence of the $U_{n}$ to the unit disk (see concluding remarks).

DEFINITION OF THE FUNCTION $h(r)$. Let $q_{r}=m_{-r} \circ \mathrm{sq} \circ m_{\sqrt{r}}$, and let $h:[0,1) \rightarrow[0,1)$ be given by

$$
h(r)=\inf \left\{|z|\left|q_{r}(z)\right|=r\right\} .
$$

Figure 3 explains the meaning of this formula. (The infimum is in fact realized by the line drawn.) Since $U_{n}$ contains the disc of radius $r=\left|a_{n}\right|, U_{n+1}$ contains the component of $q_{r}^{-1}\left(D_{r}\right)$ containing the origin, hence the disc of radius $h(r)$.

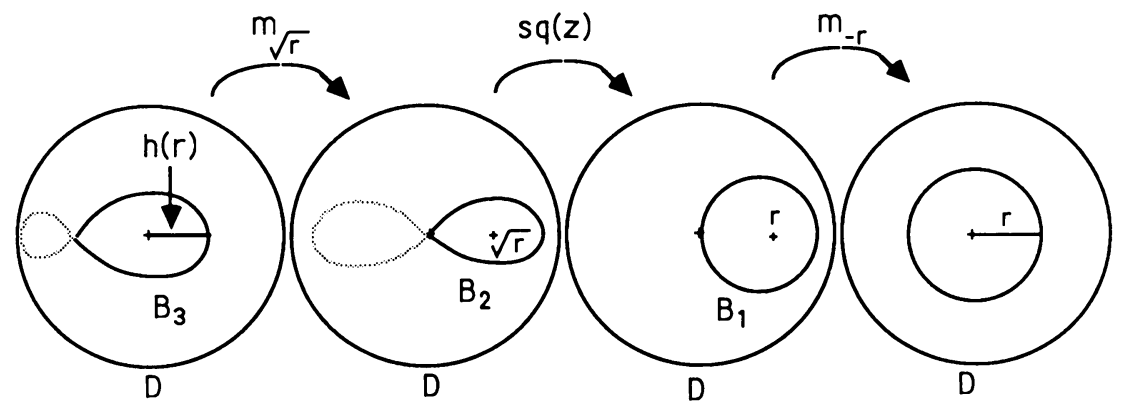

FIGURE 3

LEMMA 3.3.1. The function $h$ is continuous, and $h(r)>r$ if $r>0$.

PROOF. The function $h$ is obviously continuous. Since $q_{r}: D \rightarrow D$ with $q_{r}(0)=0$ we have $\left|q_{r}(z)\right| \leq|z|$ by Schwarz's lemma, with strict inequality if $z \neq 0$. Taking 
infimum's over $B_{r}=\left\{z|| q_{r}(z) \mid=r\right\}$, we get $h(r) \geq r$. Moreover, the set $B_{r}$ is compact, so the infimum is realized, and hence the inequality is strict unless $0 \in B_{r}$, i.e. unless $r=0$. Q.E.D.

We now show that $\left|a_{n}\right| \rightarrow 1$ using the properties of $h(r)$. By the definition of $a_{n}$ and 3.3.1 we have $\left|a_{n+1}\right| \geq h\left(\left|a_{n}\right|\right)>\left|a_{n}\right|$, so $\left\{\left|a_{n}\right|\right\}$ is strictly increasing. Let $A=\lim \left|a_{n}\right|$, and suppose $A<1$. Let $\varepsilon=h(A)-A>0$. By continuity of $h(r)$ we can choose $\delta$ such that if $|a-A|<\delta$, then $h(a)-a>\varepsilon / 2$. Now choose $N$ sufficiently large so that $\left|A-a_{N}\right|<\min (\delta, \varepsilon / 2)$. Then we have a contradiction since $A<\left|a_{n}\right|+\varepsilon / 2<h\left(\left|a_{n}\right|\right)<\left|a_{n+1}\right|$ for $n>N$. Hence $A=1$.

REMARK. It might seem that we have already proved surjectivity; after all the images of the $f_{n}$ contain larger and larger discs, and the $f_{n}$ converge. The thing that could go wrong, and which would go wrong if for instance we had chosen a divergent sequence $\tilde{0}_{n}$ of basepoints for $\tilde{U}$, is that the sequence $\left\{f_{n}\right\}$ could converge to a constant on the boundary of $D$.

PROOF OF LEMMA 3.3. We want to show that for $w \in D, w \in f(\tilde{U})$. If $w \in D$, then for some large enough $N$ there exists a $R$ such that $\left|a_{n}\right|>R>|w|$. Let $V \subset \tilde{U}$ be the component of $f_{n}^{-1}\left(D_{\left|a_{n}\right|}\right)$ containing $\tilde{0}$. The mapping $f_{n}: V \rightarrow D_{\left|a_{n}\right|}$ is a covering space and thus an isomorphism, so $V_{1}=V \cap f_{n}^{-1}\left(\bar{D}_{R}\right)$ is compact. The sets $f_{n+i}^{-1}\left(\bar{D}_{R}\right) \subset V_{1}$, because $p_{n+i} \circ \cdots \circ p_{n}$ satisfies Schwarz's lemma. Then $\left\{f_{n+i}^{-1}(w)\right\}$ is a sequence in $V_{1}$ with a limit point $v$ such that $f(v)=w$, and $w \in f(\tilde{U})$.

So $D \subset f(\tilde{U})$ and $f(\tilde{U}) \subset \bar{D}$; since $f$ is an open map $f(\tilde{U})=D$. Q.E.D.

\section{LEMMA 3.4. The mapping $f$ is a covering space of its image.}

PROOF. Let $\pi_{n}=q_{1} \circ \cdots \circ q_{n}: D \rightarrow D$, so that $p=\pi_{n} \circ f_{n}$. The sequence $\pi_{n}$ has a subsequence converging to an analytic function $\pi: D \rightarrow D$. By choosing a subsequence of the subsequence making the $f_{n}$ converge, we can guarantee that $p=\pi \circ f ;$ then $\pi(D)=\pi(f(\tilde{U}))=p(\tilde{U})=U_{0}$.

Lemma 2.4 completes the proof. The proofs of Lemma 3.4, Proposition 3.2, and the Theorem are now complete.

4. Concluding remarks. (1) Since $\tilde{U}$ is isomorphic with $D$, we see that $\pi$ is a universal covering map for $U$. It can be used to explicitly estimate the Poincaré metric in $U$.

(2) We can make the $f_{n}$ and $\pi_{n}$ converge (not just via a subsequence) by normalizing their derivatives to be positive. However, this is not necessary in practice where some $\pi_{n}$ is chosen to approximate a universal cover of $U$, and an arbitrary rotation composed with $\pi_{n}$ is not important.

(3) If only Case 1 occurs, which is the case if and only if $U$ is simply connected, the above gives a proof of the Riemann mapping theorem due to Koebe. The map $\pi: D \rightarrow U$ is a conformal isomorphism.

(4) The sequence $\left\{\left|a_{n}\right|\right\}$ is actually bounded below by $\left\{h^{\circ} n\left(\left|a_{1}\right|\right)\right\}$, so examining $\left\{h^{\circ} n\left(\left|a_{1}\right|\right)\right\}$ gives a worst case behavior for the rate of convergence of the algorithm.

The square root of a circle through the origin is lemniscate (this is the boundary of $B_{2}$ in Figure 3 ), and we can compute the minimum distance from 0 to $m_{-\operatorname{sqrt}(r)}\left(\partial B_{2}\right)=B_{3}$. If $r$ is the radius of the original disk, then the minimum 
distance from 0 to the boundary $q_{r}^{-1}\left(D_{r}\right)$ is

$$
\begin{aligned}
& h(r)=\frac{\sqrt{2 r}-\sqrt{r^{3}+r}}{\sqrt{r^{2}+1}-\sqrt{2 r}}=\frac{\sqrt{r}\left(r-1+\sqrt{2 r^{2}+2}\right)}{(r+1)}, \\
& h(r)=1+(r-1)-\frac{1}{16}(r-1)^{3}+\cdots .
\end{aligned}
$$

It is simple to check that $h$ has a fixed point at 1 which attracts all $r \in(0,1)$ under iteration. We can estimate the rate of convergence of $h^{\circ} n(r)$ to $1-\varepsilon$.

In fact,

$$
h^{\circ} n(r)=1-2 \sqrt{2} / \sqrt{n}+o(1 / \sqrt{n}) .
$$

For a more practical estimate, suppose $1 / 2<r<1$. If $h^{\circ} n(r)=1-\varepsilon$, then $n$ satisfies

$$
(4 / \varepsilon)^{2}-(2 /(1-r))^{2}>n>(2 / \varepsilon)^{2}-(4 /(1-r))^{2} .
$$

This estimate follows by induction from the fact that if $2 / \sqrt{m}<1-r<4 / \sqrt{m}$, then $2 / \sqrt{m+1}<1-h(r)<4 / \sqrt{m+1}$, which follows from the asymptotic expansion for $h^{\circ} n(r)$ above. This is not nearly a sharp estimate. The inequalities can be improved by requiring $r$ to be closer to 1 .

When $n$ is large enough so that $1-\varepsilon<h^{\circ} n\left(\left|a_{1}\right|\right)$, then $1-\varepsilon<\left|a_{n}\right|$, and $\rho_{U_{n}}(z)$, the Poincaré metric for $U_{n}$, can be computed to within $\varepsilon$ by approximating $U_{n}$ as $D$; for in that case

$$
\rho_{D}(0) \leq \rho_{U_{n}}(0) \leq(1-\varepsilon) \rho_{D}(0) \text {. }
$$

\section{REFERENCES}

[A] L. V. Ahlfors, Complex analysis, McGraw-Hill, New York, 1979.

[B] P. Blanchard, Complex analytic dynamics on the Riemann sphere, Bull. Amer. Math. Soc. (N.S.) 11 (1984), 85-141.

[F] O. Forster, Lectures on Riemann surfaces, Springer-Verlag, New York, 1981.

Department of Mathematics, Cornell University, IthaCA, NeW York 14853 